\title{
Ecodistribution des tabanidés, glossines et stomoxes le long d'un transect forêt primaire -village au Gabon
}

\author{
Christophe Roland ZINGA KOUMBA ${ }^{1,3,4^{*}}$, Ornella Anaïs MBANG NGUEMA ${ }^{1,2}$, \\ Jacques François MAVOUNGOU ${ }^{1,2}$ et Piazzy OBAME ONDO KUTOMY ${ }^{2}$ \\ ${ }^{1}$ Institut de Recherche en Ecologie Tropicale (IRET), BP 13354, Libreville, Gabon. \\ ${ }^{2}$ Université des Sciences et Techniques de Masuku, BP 941, Franceville, Gabon. \\ ${ }^{3}$ Ecole Régionale Post-Universitaire d'Aménagement et de gestion Intégrés des Forêts \\ et Territoires Tropicaux (ERAIFT), BP 15373, Kinshasa, R. D. Congo. \\ ${ }^{4}$ Ecole Régionale Post-Universitaire d'Aménagement et de Gestion Intégrées des Forêts \\ et Territoires Tropicaux(ERAIFT). Université de Kinshasa, Commune de Lemba-Kinshasa, \\ République Démographique du Congo. BP 13 354, Tel : +241 04404923. \\ *Corresponding author; E-mail: zinga.koumba@yahoo.fr
}

\section{RESUME}

L'abondance et la diversité spécifique des mouches hématophages ont été évaluées par des captures d'insectes à l'aide des pièges Vavoua et Nzi durant la saison sèche et la saison des pluies dans deux types de biotope au Gabon : Forêt primaire et village. Trois groupes d'intérêt médical et vétérinaire ont été ciblés pour cette étude : les tabanidés, les glossines et les stomoxes. Au total, 21 espèces de mouches hématophages ont été identifiées: treize espèces de Tabanidae, cinq espèces de Glossinidae et trois espèces de Stomoxyinae. Les espèces de tabanidés numériquement les plus abondantes ont été : Chrysops. silacea (19\%), Tabanus obscurehirtus (13\%), T. taeniola (12\%), T. dilitius (9\%), T. par (7\%), T. socius (7\%), T. obscurefumalus (7\%), T. canus (7\%), T. boueti (7\%), T. marmorosus congoicola (6\%). Les espèces les moins abondantes ont été $T$. secedens $(2 \%)$, C. dimidiatus $(2 \%)$ et $A$. fasciata $(2 \%)$. Les espèces de glossines les plus abondantes ont été Glossina nashi (33\%) et G. palpalis palpalis (33\%). Les moins abondantes ont été G. frezili (15\%), G. fusca congolensis (14\%) et G. tabaniformis (5\%). Les espèces de stomoxes ont été représentées par Stomoxys omega (43\%), S. niger niger (43\%) et S. niger bilineatus (14\%).

(C) 2014 International Formulae Group. All rights reserved.

Mots clés : Insectes hématophages, Nzi, Vavoua, Zadié, Gabon.

\section{INTRODUCTION}

La connaissance des insectes hématophages est d'une grande importance en médecine humaine et vétérinaire (Leclercq, 1967 ; Muzari et al., 2010). Les diptères hématophages, en l'occurrence tabanidés, glossines et stomoxes, de par leur comportement alimentaire et la nature de leurs pièces buccales, sont des vecteurs mécaniques et/ou biologiques de plusieurs agents pathogènes (Oldroyd, 1954 ; Zumpt, 1973 ; Koné et al., 2011 ; Baldacchino et al., 2013). Certains, tels que tabanidés et stomoxes sont impliqués dans la transmission mécanique des trypanosomes (Rodhain and Perez, 1985; Foil, 1989 ; Baldacchino et al., 2013) et de la 
filariose à Loa loa. (Van Hoegaerden et al., 1987 ; Touré et al., 1998, 1999 ; Koné et al., 2011). Tandis que les glossines constituent les principaux vecteurs de la Trypanosomose Humaine Africaine (THA) ou maladie du sommeil et de la Trypanosomose Animale Africaine (TAA).

$\mathrm{Au}$ Gabon, les connaissances qui entourent les insectes hématophages demeurent encore fragmentaires et peu documentées (Laveissière, 1985 ; Mavoungou et al., 2008, 2012). Aucune information relative aux tabanidés, glossines et stomoxes du village de Zadié attenant à la forêt primaire, située dans un foyer «quiescent » de la maladie du sommeil (Geogelin, 1923; Milleliri et al., 2009) et dans une zone endémique de loase, n'est disponible de nos jours. Pourtant, ces insectes peuvent représenter un risque pour les populations humaines et animales par leur nuisance directe, mais également par leur rôle de vecteur. Par ailleurs, la meilleure stratégie de contrôle de ces insectes passe nécessairement par la connaissance de la distribution et de l'abondance des différentes espèces vectrices de pathogènes. Aussi, la connaissance sur la distribution et l'abondance des vecteurs potentiels est un préalable à la compréhension $\mathrm{du}$ fonctionnement de tout système vectoriel. Nous présentons ici des données préliminaires sur l'éco-distribution des tabanidés, glossines et stomoxes réalisées dans deux biotopes différents : le village Zadié et la forêt primaire attenant à ce village.

\section{MATERIEL ET METHODES \\ Site d'étude}

Le site d'étude est situé dans le nord-est du Gabon, dans la province de Ogooué Ivindo, à $50 \mathrm{~km}$ de la ville de Makokou (Figure 1). Les sessions de piégeages se sont déroulées dans le village Zadié (milieu anthropisé) et dans la forêt primaire (milieu non anthropisé) gérées durablement, par l'entreprise Olam, dans la partie sud du bloc Monts Belinga, près de la rivière Zadié $\left(0^{\circ} 57^{\prime} \mathrm{N}, \quad 13^{\circ} 10^{\prime} \mathrm{E}\right)$. Les précipitations annuelles moyennes varient entre 1600 et $1700 \mathrm{~mm}$ avec deux saisons sèches (juin-août et janvier-mars) et deux saisons des pluies (avril-mai et septembre-décembre). La température moyenne annuelle est de $23,9{ }^{\circ} \mathrm{C}$. Le relief est constitué d'un plateau vallonné composé de nombreuses collines, à une altitude d'environ $500 \mathrm{~m}$, sur un socle cristallin archéen. Les sols sont des sols ferralitiques jaunes peu profonds. $\mathrm{La}$ végétation appartient à la forêt dense de la région guinéo-congolaise, avec une abondance de Scyphocephalium mannii, Pycnanthus angolensis, Pentaclethra eetveldeana, Celtis sp., Gilletiodendron pierreanum, Gilbertiodendron dewevrei (TEREA, 2010).

\section{Capture des mouches hématophages}

Deux types de pièges ont été utilisés dans cette étude: les pièges Vavoua et Nzi (Laveissière \& Grébaut, 1990 ; Mihok, 2002 ; Mavoungou, 2008 ; Mavoungou et al., 2012).

Le piégeage a consisté en un réseau de 24 pièges dont 12 Vavoua et $12 \mathrm{Nzi}$, placés aux mêmes points identifiés au préalable le long d'un transect d'environ $12 \mathrm{~km}$, suivant un gradient d'anthropisation allant de la forêt primaire jusqu'au village Zadié.

Suivant l'importance de la zone prospectée, 8 à 16 pièges ont été utilisés pour chaque biotope. Ainsi, un total de huit pièges dont quatre Vavoua et quatre Nzi ont été posés en milieu anthropisé en respectant la même séquence 1 Vavoua et $1 \mathrm{Nzi}$ par point de piégeage et une distance d'au moins $500 \mathrm{~m}$ entre chaque piège. Tandis qu'en forêt primaire, 16 pièges dont huit Vavoua et huit Nzi ont été posés toujours en respectant la même séquence comme en milieu anthropisé.

Toutes les captures ont été réalisées entre avril et mai 2012, c'est-à-dire pendant la grande saison des pluies; et entre août et septembre 2012 période de grande saison sèche. La durée de piégeage a été de 20 jours consécutifs par saison. Soit 40 jours de piégeage durant toute la session de piégeage. Tous les points de capture ont été géo référencés à l'aide d'un GPS de marque GARMIN. Les pièges ont été activés le matin avant 7 heures et relevés le soir après 17 heures. Lors de la relève des pièges, les cages de capture ont été étiquetées avec le numéro 
du piège et ramenées au laboratoire pour identification.

\section{Identification des insectes}

Les insectes ont été triés au fur et à mesure de leur arrivée au laboratoire. Une première séparation était faite entre les genres stomoxes, tabanidés et glossines et les autres insectes sous une loupe binoculaire. La discrimination entre les différentes espèces de stomoxes a été faite à l'aide de la clé de Zumpt (1973), et du caractère morphologique complémentaire décrit par Garros et al. (2004) pour mieux séparer $S$. calcitrans et $S$. niger. L'identification des différentes espèces de tabanidés a été faite à l'aide des clés publiées par Oldroyd (1952, 1954, 1957, 1973). Quant aux glossines, les différentes espèces ont été identifiées sur la base des critères morphologiques (coloration des tarses et de l'abdomen) et de la structure des génitalia (Pollock, 1982 ; Brunhes et al., 1988).

\section{Analyse des données}

L'abondance de chacun de ces insectes hématophages étudiés est traduite par sa densité apparente par piège et par jour (DAP) calculée selon la formule suivante :

$$
\text { DAP }=\frac{\text { Nombre de mouches capturées }}{\text { nombre de pièges } x \text { nombre de jours de capture }}
$$

L'indice de diversité de Shannon, qui permet de quantifier l'hétérogénéité de la biodiversité d'un milieu, a été calculé suivant la formule suivante :

$$
H^{\prime}=-\sum_{i=1}^{S} p i \log _{\mathbf{2}} p i
$$

Où " $\mathrm{H}$ "' est l'indice de biodiversité de Shannon; " $i$ "'une espèce du milieu d'étude et " $p i$ ' 'la proportion d'une espèce $\mathrm{i}$ par rapport au nombre total d'espèces de mouches étudiées (S) dans le milieu étudié.

Le test du Chi carré $\left(\mathrm{X}^{2}\right)$ a été effectué pour comparer la distribution des différentes espèces étudiées selon les saisons et les biotopes.

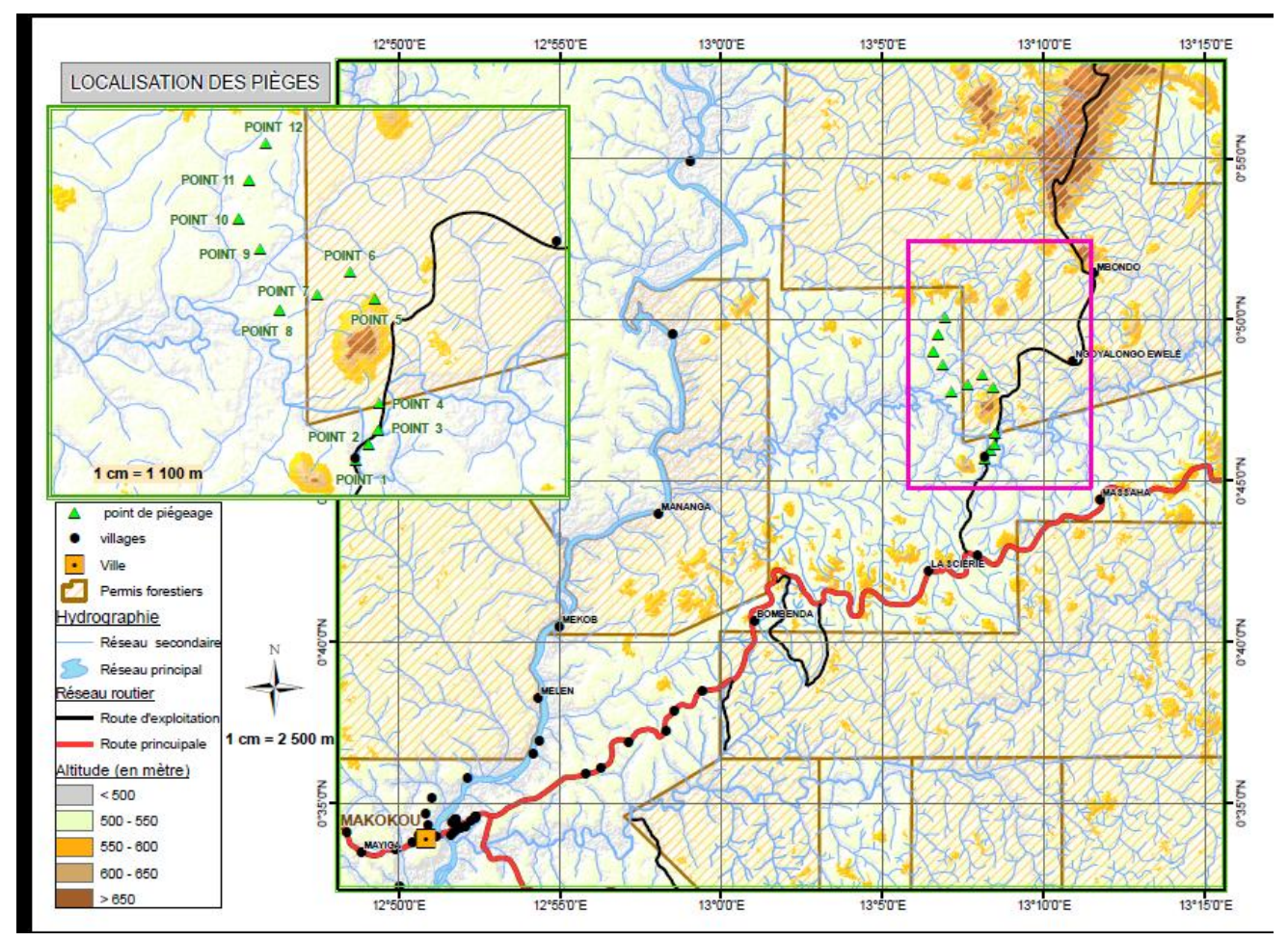

Figure 1 : Localisation de la zone d'étude. 


\section{RESULTATS}

Au total, 1500 mouches hématophages ont été capturées dont $1116(74,4 \%)$ en forêt primaire et $384(25,6 \%)$ au village. Pendant toute la session de piégeage, 21 espèces ont été identifiées (Tableau 1) : soit 13 espèces de Tabanidae, 5 espèces de Glossinidae et 3 espèces de Stomoxyinae.

\section{Composition des populations de Tabanidae capturées}

Au total, 623 tabanidés ont été récoltés en 40 jours de piégeage, soit une densité apparente par piège (DAP) de 1,29 tabanidé/piège/jour.

\section{Composition par genre}

Dans les deux biotopes, le genre Tabanus a été le plus abondant $(75,6 \%)$ suivi du genre Chrysops (22,2\%). Le genre Ancala a été très faiblement représenté avec $(2,2 \%)$. En forêt primaire, le genre Tabanus a été le genre le plus représenté avec un taux de 93,3\% ; alors qu'au village c'est le genre Chrysops qui a été le plus représenté $(64,1 \%)$ (Figure 2).

\section{Composition par espèce}

Dix espèces du genre Tabanus ont été identifiées: Tabanus obscurehirtus (13\%), T. taeniola (12\%), T. dilitius (9\%), T. par (7\%), T. socius (7\%), T. obscurefumatus (7\%), $T$. canus (7\%), T. boueti (7\%), T. marmorosus congoicola (6\%), T. secedens (2\%) (Figure 3).

Le genre Chrysops comprenait deux espèces: Chrysops dimidiatus (2\%) et $C$. silacea (19\%). Alors que le genre Ancala était représenté par une seule espèce : A. fasciata $(2 \%)$.

\section{Abondance relative des tabanidés en fonction des biotopes étudiés}

Le maximum des captures a été observé en forêt primaire $(70 \%)$ et le minimum au village (30\%). Les indices de diversité de Shannon, calculés pour chaque milieu ou station de piégeage, permettent de donner une idée de la répartition des espèces en fonction de biotope. La forêt primaire présente la diversité la plus importante avec un indice de diversité de 2,37. Au village, cet indice est de 1,28 .

En forêt primaire, on note une forte richesse spécifique caractérisée par la présence de 13 espèces : T. taeniola (16\%), $T$. dilitius (13\%), T. obscurehirtus (12\%), T.canus (10\%), T. boueti (10\%), T. socius (10\%), T. obscurefumatus (10\%), T.par (8\%), T. marmorosus congoicola (3\%), T. secedens (3\%), A. fasciata (3\%), C. dimidiatus (2\%) et C.silacea (2\%).

Dans le village, huit espèces ont été capturées : C. silacea (62\%), T. obscurehirtus (13\%), T. marmorosus congoicola (10\%), T. par (6\%), T. taeniola (4\%), T. boueti (2\%), C. dimidiatus (2\%) et $T$. socius (1\%).C. silacea a présenté une forte abondance au village mais était très faible en forêt primaire. Il est à noter que les huit espèces capturées au village sont aussi présentées en forêt primaire avec une abondance variable.

Le résultat du test du $\chi^{2}$ a révélé une différence hautement significative au seuil de 0,001 p 100 dans la distribution des différentes espèces selon le biotope. $\left(\chi^{2}=\right.$ $366,55 ; \mathrm{ddl}=12 ; \mathrm{p}<0,001)$.

\section{Abondance relative des tabanidés en fonction des saisons}

$\mathrm{Au}$ village, six espèces de Tabanidés ont été identifiées en saison des pluies: C.silacea (57\%), T. obscurehirtus (18\%), T. marmorosus congoicola (14\%), T. par (7\%), T. boueti (2\%) et T. taeniola (2\%). Alors qu'en saison sèche, cinq espèces ont été capturées: C. silacea (76\%), C. dimidiatus (8\%), T. taeniola (8\%), T.par (4\%) et T. socius (4\%). On constate que Chrysops 
silacea et Tabanus taeniola ont été capturées durant les deux saisons.

En forêt primaire, 12 (douze) espèces de tabanidés ont été capturées en saison des pluies: T. obscurehirtus (16\%), T. dilitius (14\%), T. taeniola (14\%), T. canus (10\%), T.boueti (10\%), T. socius (10\%), T. obscurefumalus (9\%), T. par (6\%), T. marmorosus congoicola (4\%), Ancala fasciata (4\%), T. secedens (3\%) et Chrysops silacea (1\%). Tandis qu'en saison sèche, neuf espèces ont été identifiées : T.par (26\%), T. taeniola (23\%), C. dimidiatus (19\%), T. obscurefumalus (14\%), C. silacea (9\%), T. boueti (2\%), T. socius (2\%), T. obscurehirtus (2\%) et $T$. socius $(2 \%)$. On remarque que Chrysops dimidiatus n'a pas été capturé en saison des pluies.

Le résultat du test du $\chi^{2}$ a révélé une différence hautement significative au seuil de 0,001 p. 100 dans la distribution des différentes espèces de tabanide selon la saison $(\chi 2=111,75 ; \mathrm{ddl}=9 ; \mathrm{p}<0,001)$.

\section{Composition des populations de Glossinidae capturées}

Au total, 564 glossines ont été récoltées en 480 piège-jours, soit une densité apparente par piège (DAP) de 1,2 G/P/J. Sur ce total, $437(77,48 \%)$ spécimens ont été capturés en forêt primaire et $127(22,52 \%)$ au village. Ces résultats correspondent à des DAP de 0,91 $\mathrm{G} / \mathrm{P} / \mathrm{J}$ en forêt primaire et de $0,26 \mathrm{G} / \mathrm{P} / \mathrm{J}$ au village.

\section{Composition par espèces}

Cinq espèces et sous-espèces de glossines appartenant au sous-genre Austenina (67\%) et au sous-genre Nemorhina (33\%) ont été capturées.

Le sous-genre Austenina comprenait quatre espèces (Figure 4): Glossina nashi (33\%), Glossina frezili (15\%), Glossina fusca congolensis (14\%) et Glossina tabaniformis $(5 \%)$.

Le sous-genre Nemorhina comprenait une seule espèce: Glossina palpalis palpalis $(33 \%)$.

\section{Abondance relative des glossines en fonction des biotopes}

Le maximum de capture a été observé en forêt primaire $(77 \%)$ et le minimum au village (23\%). La diversité du peuplement des glossines dans les deux biotopes a été déterminée par l'indice de Shannon. Cet indice est de 1,46 en forêt primaire et de 1,04 au village.

En forêt primaire, cinq espèces ont été capturées. Il s'agit de G.nashi, G. palpalis palpalis, G. frezili, G. fusca et $G$. tabaniformis. G. nashi a été la mieux représentée avec $37 \%$, suivi de G. palpalis palpalis (27\%). G. frezili (19\%) et G. fusca congolensis $(13 \%)$ ont été moins abondantes. G. tabaniformis $(6 \%)$ a été très faiblement représentée.

$\mathrm{Au}$ village, quatre espèces ont été identifiées: G. nashi, G. palpalis palpalis, G. frezili et $G$. fusca congolensis avec des abondances différentes (Figure 5). G. palpalis palpalis (58\%) a été l'espèce la plus abondante suivi par G. nashi $(20 \%)$ et $G$. fusca congolensis (19\%). G. frezili (2\%) a été l'espèce la moins abondante.

Le résultat du test du $\chi^{2}$ a révélé une différence hautement significative au seuil de 0,001 p 100 dans la distribution des différentes espèces selon le biotope $(\chi 2=$ $67,32 ; \mathrm{ddl}=4 ; \mathrm{p}<0,001)$.

\section{Abondance relative des glossines en fonction de la saison}

$\mathrm{Au}$ village, le maximum de capture a été observé en saison des pluies (53\%) et le minimum en saison sèche $(47 \%)$. Trois espèces de glossines ont été identifiées en 
saison des pluies : G. palpalis palpalis $(69 \%)$, G. fusca congolensis (16\%) et G. nashi (15\%). Tandis qu'en saison sèche, quatre espèces ont été capturées : G. palpalis palpalis (47\%), G. nashi (27\%), G. fusca congolensis (22\%) et $G$. frezili $(5 \%)$. Toutes les espèces capturées en saison des pluies ont été capturées aussi en saison sèche avec des abondances différentes. Cependant, on note que G. frezili n'a été capturée qu'en saison sèche avec une très faible abondance.

Les indices de diversité observés en saison des pluies et en saison sèche sont respectivement de 0,83 et de 1,18 . Le résultat du test du $\chi 2$ a révélé qu'il n'existe pas de différence significative dans la distribution des différentes espèces selon la saison $(\chi 2=$ 8,5699; ddl = 3; p>0,001).

En forêt primaire, le maximum de captures a été effectué en saison des pluies (74\%) et le minimum en saison sèche $(36 \%)$. Cinq espèces de glossines ont été identifiées en saison des pluies. Il s'agit de G. nashi (33\%), G. palpalis palpalis (28\%), G. frezili (16\%), G. fusca congolensis (14\%) et $G$. tabaniformis (8\%). Alors qu'en saison sèche quatre espèces ont été capturées: G. nashi (48\%), G. palpalis palpalis (19\%), G. frezili (24\%) et G. fusca congolensis. Il est à noter que G. nashi et G. palpalis palpalis ont été les espèces les plus abondantes durant les deux saisons.

Les indices de Shannon observé en saison des pluies et saison sèche sont respectivement de 1,50 et 1,22 . Le résultat du test du $\chi 2$ a révélé une différence hautement significative au seuil de 0,001 p 100 dans la distribution des différentes espèces selon le biotope $\left(\chi^{2}=21,639 ; \mathrm{ddl}=4 ; \mathrm{p}<0,001\right)$.

\section{Composition des populations de Stomoxyinae capturées}

Un total de 313 stomoxes a été capturé dont $65(20,8 \%)$ au village et $248(79,2 \%)$ en forêt primaire. Ce total correspondant à une densité apparente par piège (DAP) et par jour de 0,65 stomoxe/piège/jour.

\section{Composition par espèces}

Trois espèces de stomoxes ont été capturées durant la période d'étude : Stomoxys omega (43\%), Stomoxys niger niger (43\%) et Stomoxys niger bilineatus (14\%). S. omega et $S$. niger niger ont constitué les espèces les plus abondantes. S. niger bilineatus a été l'espèce la moins représentée (Figure 6).

\section{Abondance relative des stomoxes en fonction des biotopes}

Le maximum des captures a été observé en forêt primaire $(79 \%)$ et le minimum au village (20\%) (Figure 7). L'indice de diversité Shannon a été de 1,02 en forêt primaire et de 0,54 au village.

En forêt primaire trois espèces ont été identifiées : S. omega (48\%), S. niger niger $(34 \%)$ et $S$. niger bilineatus (17\%). Au village, deux espèces ont été capturées : $S$. niger niger (77\%) et $S$. omega (23\%). Toutes les espèces capturées au village ont été capturées également en forêt primaire avec une abondance différente. On note une forte abondance de $S$. niger niger au village (Figure 8).

La diversité du peuplement des stomoxes dans les deux biotopes a été déterminée par l'indice de Shannon. Elle a montré que le maximum d'espèces a été capturé en forêt primaire avec un indice de 0,62 et le minimum au village $\left(H^{\prime}=0,35\right)$. Ainsi, le résultat du test du $\chi 2$ a révélé une différence hautement significative au seuil de 0,001 p 100 dans la distribution des différentes espèces selon le biotope $\left(\chi^{2}=\right.$ 40,639; $\mathrm{ddl}=2 ; \mathrm{p}<0,001)$.

\section{Abondance relative des stomoxes en fonction des saisons}

Au village, le maximum des captures a été observé en saison des pluies $(77 \%)$ et le 
minimum en saison sèche (23\%). En saison sèche tout comme en saison des pluies deux espèces ont été capturées. Il s'agit de $S$. niger niger et $S$. omega. En saison sèche, S. niger niger et $S$. omega ont présenté une abondance respectivement de $80 \%$ et $20 \%$. Tandis qu'en saison des pluies $S$. niger niger a présenté une abondance de $76 \%$ et $S$. omega une abondance de $24 \%$. La richesse spécifique de ces espèces exprimée par l'indice Shannon est presque uniforme durant les deux saisons : $\mathrm{H}^{\prime}=0,6$ en saison des pluies et $\mathrm{H}^{\prime}=0,5$ en saison sèche. Le résultat du test du $\chi 2$ a révélé qu'il n'existe pas de différence significative dans la distribution des différentes espèces selon la saison $(\chi 2=0,104 ; \mathrm{ddl}=1 ; \mathrm{p}>0,001)$.

En forêt primaire, 173 stomoxes ont été capturés en saison des pluies et 75 en saison sèche. Ces stomoxes se répartissent en trois espèces. Il s'agit de S. niger niger, S. omega et $S$. niger bilineatus. En saison des pluies, $S$. omega présentait la plus forte abondance (50\%) suivi de S. niger niger $32 \%$. S. niger bilineatus est faiblement représenté (18\%). En saison sèche, $S$. niger niger avait une abondance de $45 \%$, S. omega $39 \%$ et S. niger bilineatus $16 \%$. S. bilineatus a été l'espèce la moins abondante durant les deux saisons. La richesse spécifique de ces espèces exprimée par l'indice Shannon est presque uniforme durant les deux saisons : $H^{\prime}=1,021$ en saison des pluies et $H^{\prime}=1,019$ en saison sèche. Aussi, le résultat du test du $\chi 2$ a révélé qu'il n'existe pas de différence significative dans la distribution des différentes espèces selon la saison $(\chi 2=0,923 ; \mathrm{ddl}=2 ; \mathrm{p}>0,001)$.

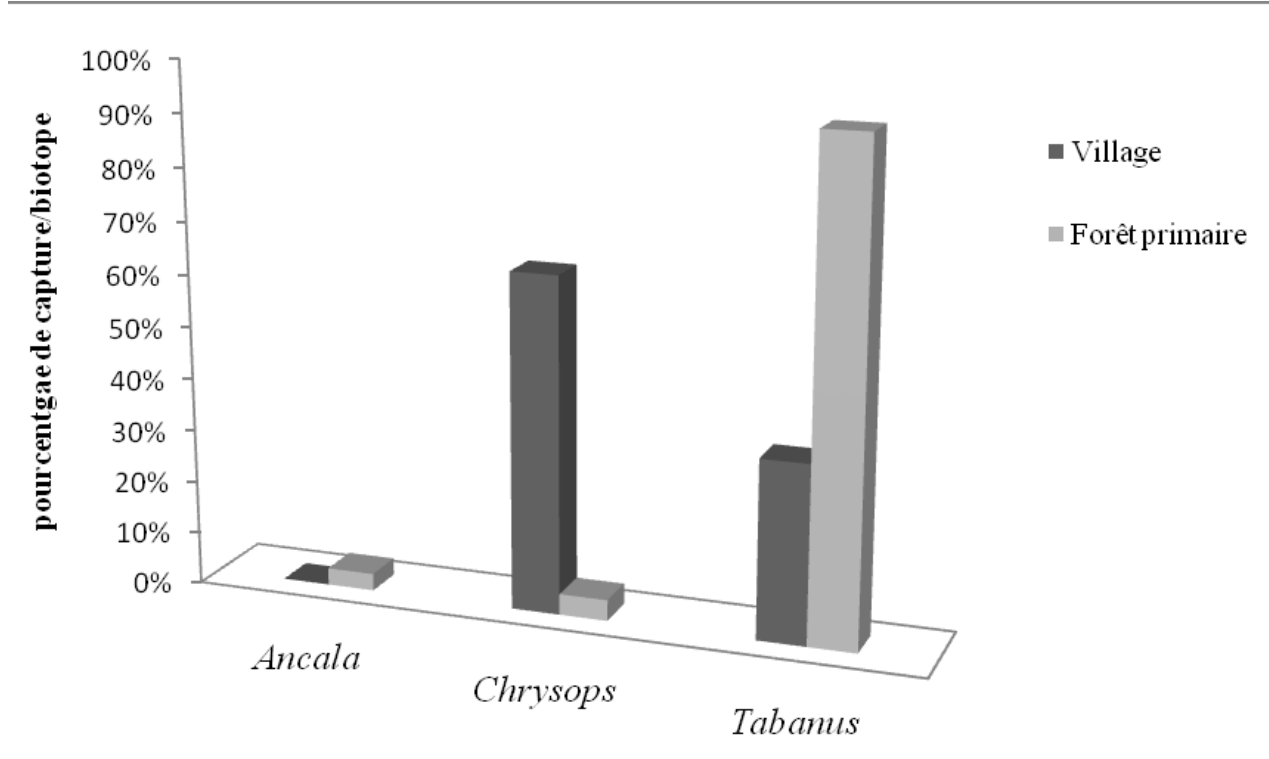

Genres

Figure 2 : Répartition des genres de tabanidés capturés en fonction des biotopes prospectés. 


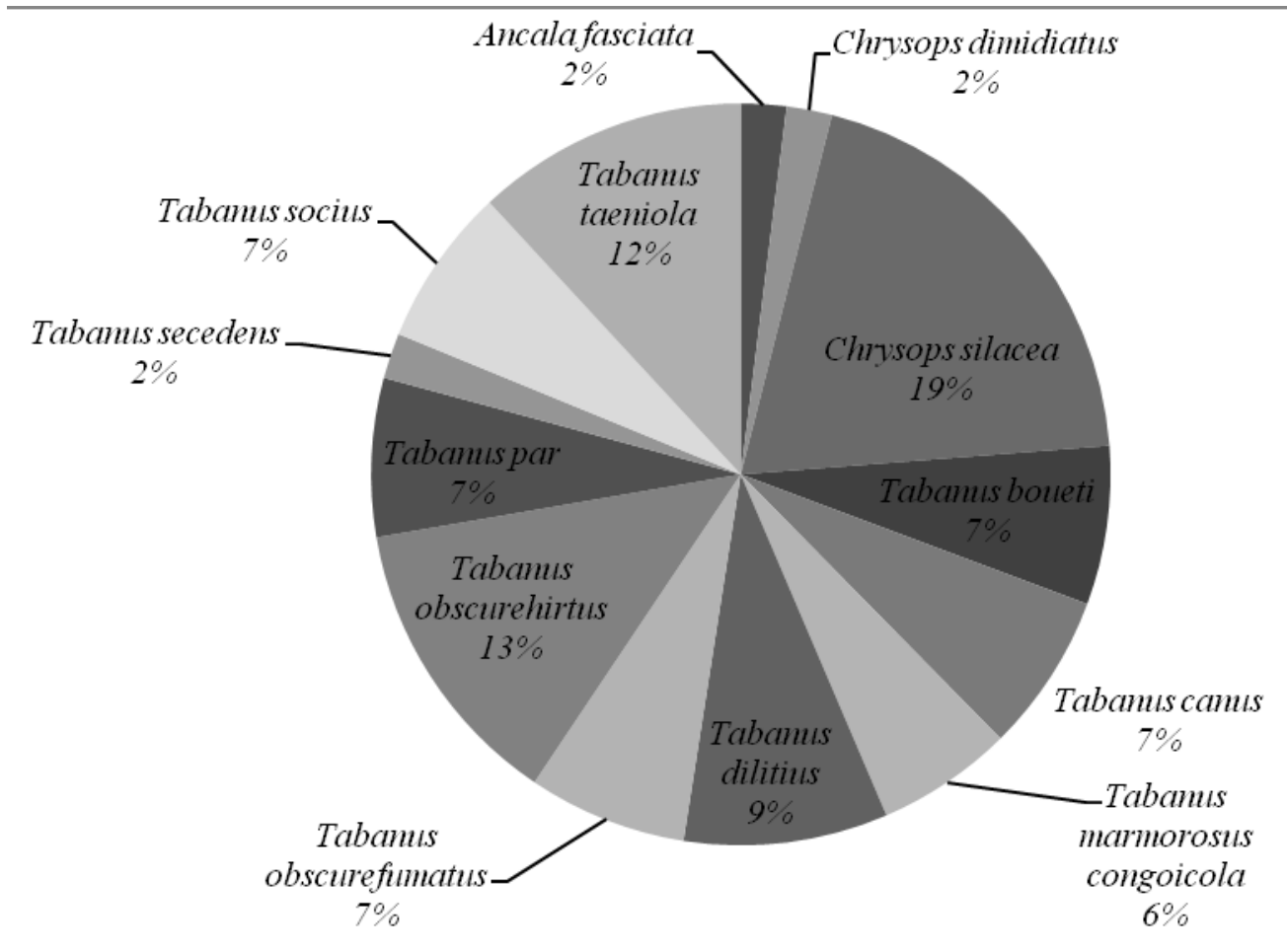

Figure 3 : Répartition spécifique des espèces de tabanidés capturés.

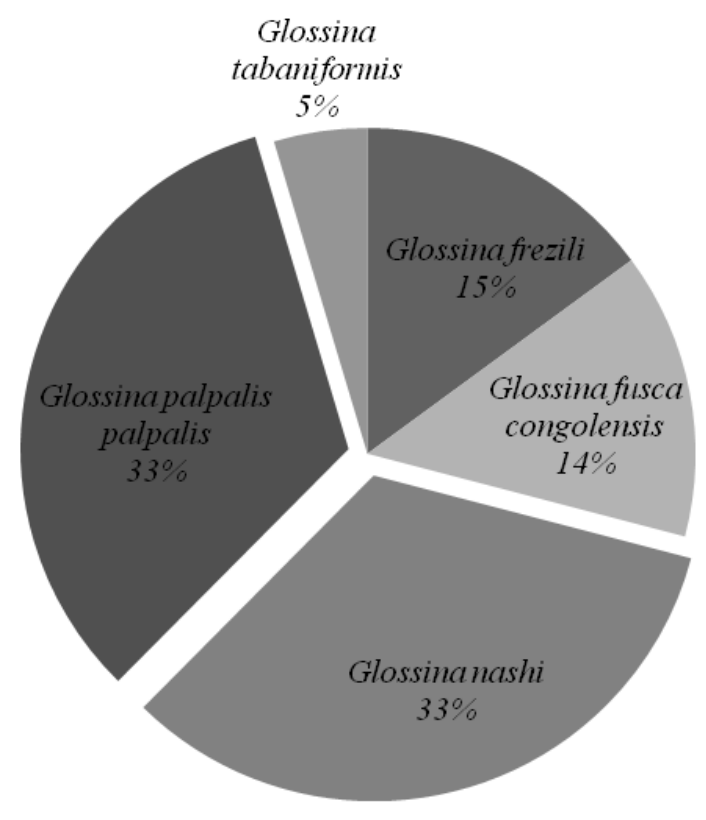

Figure 4 : Répartition et abondance spécifique des espèces de Glossines capturées. 


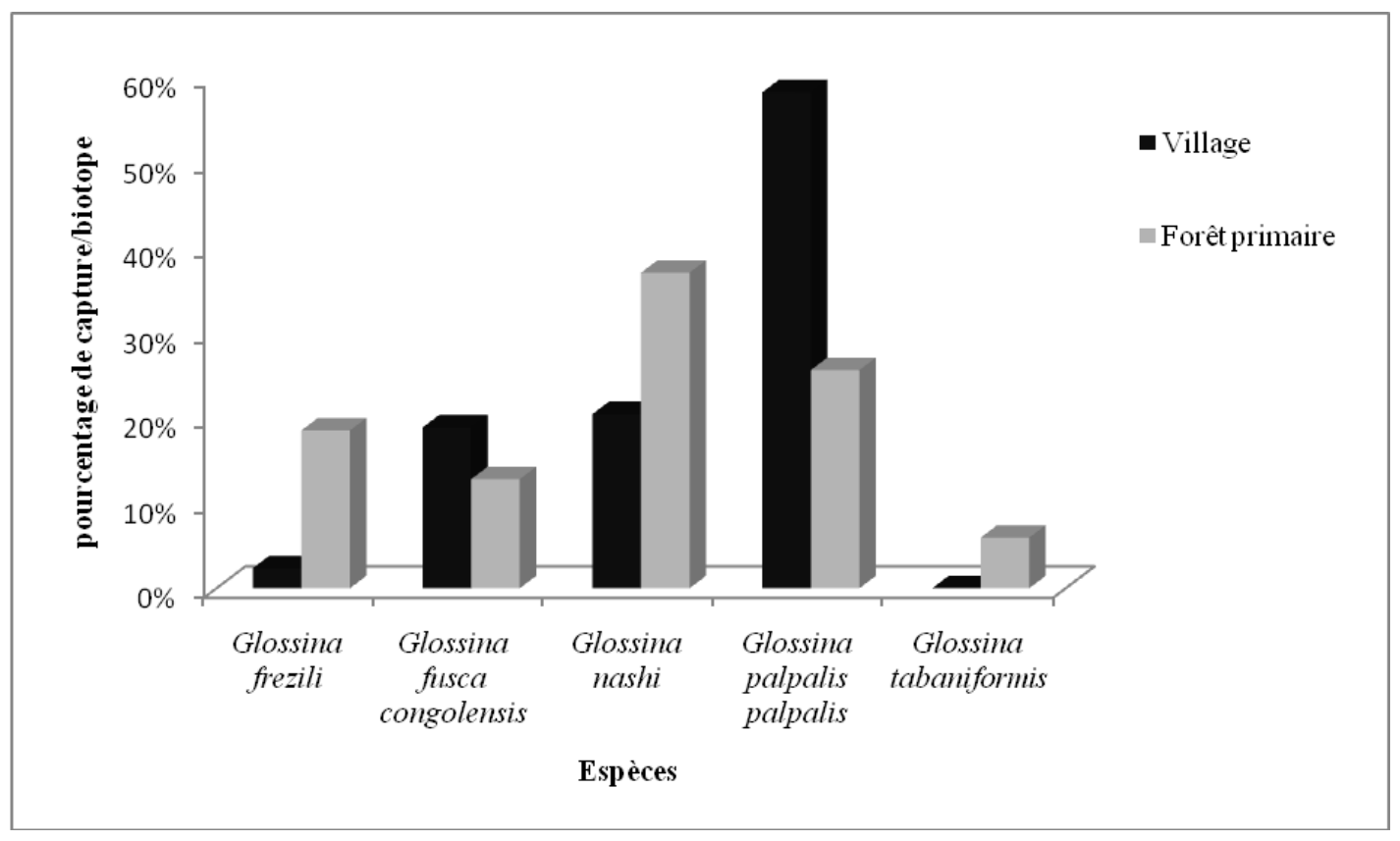

Figure 5 : Répartition des espèces de glossines capturées en fonction des biotopes prospectés.

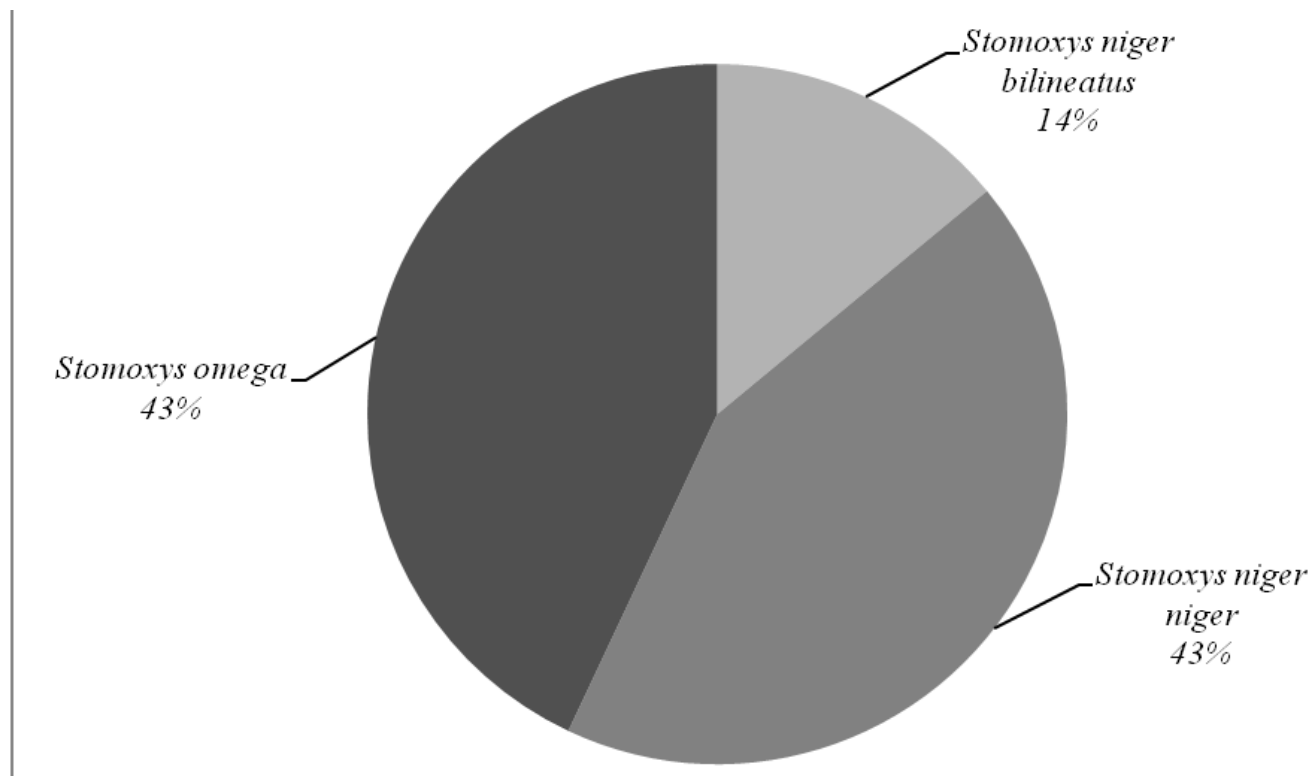

Figure 6 : Répartition spécifique des espèces de stomoxes capturées. 


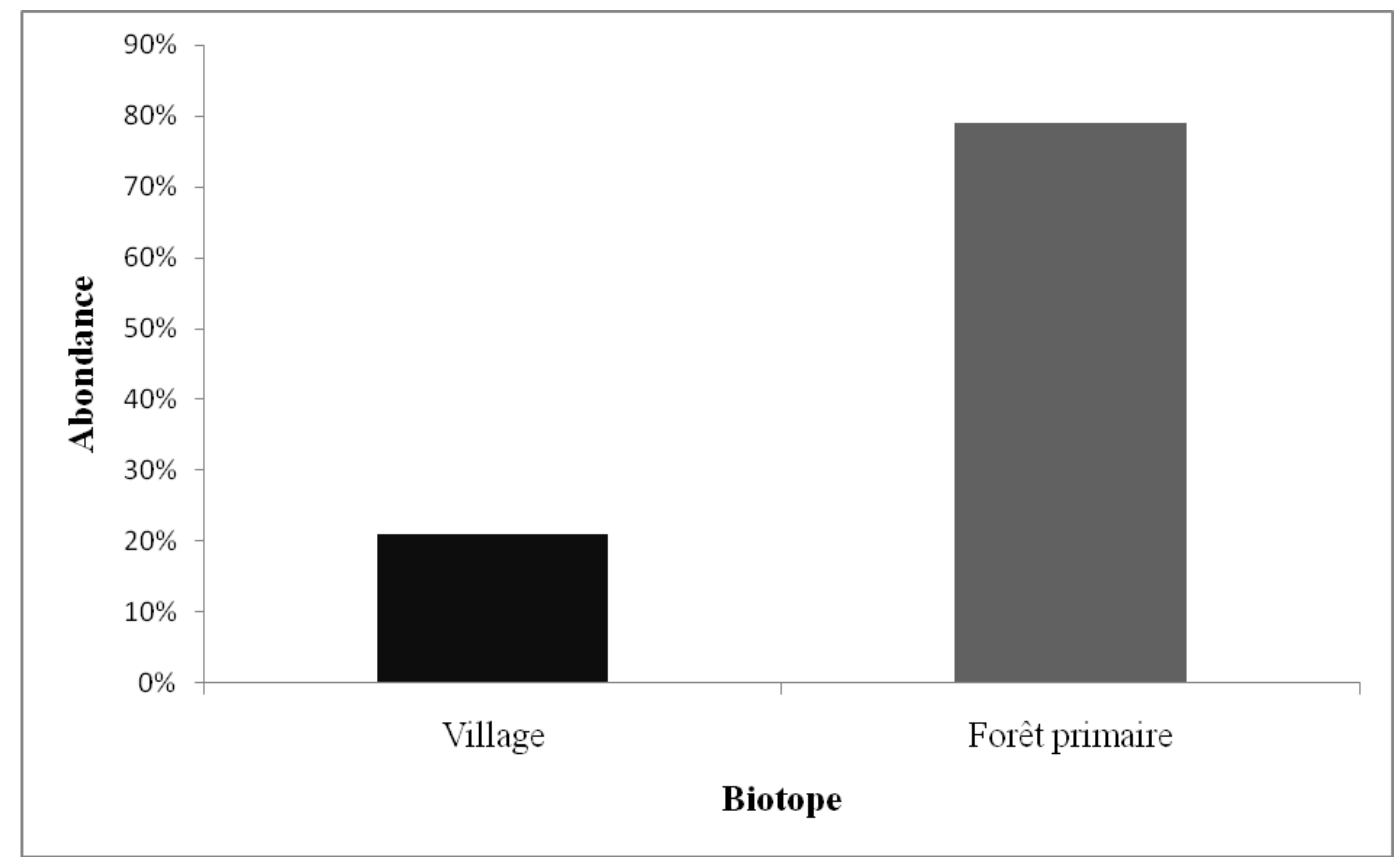

Figure 7 : Abondance des stomoxes capturés selon le biotope.

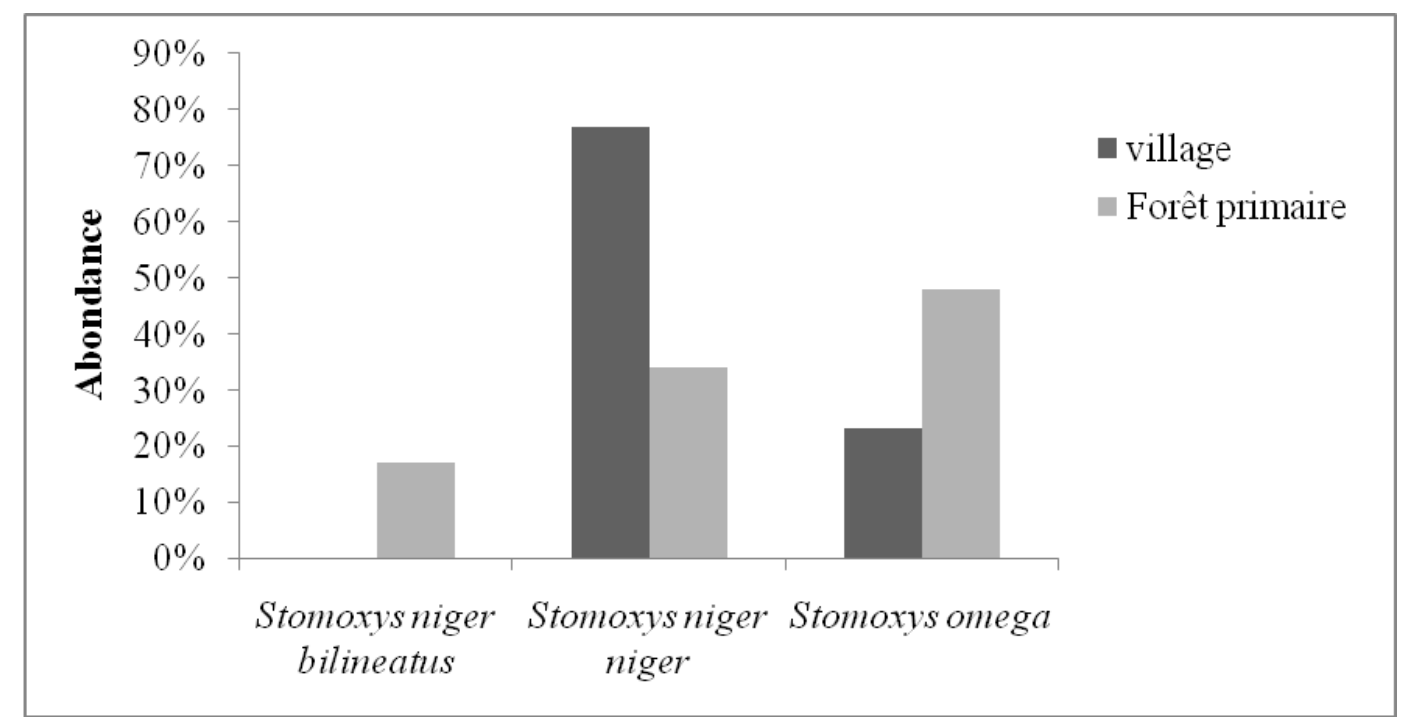

Figure 8 : Répartition spécifique des espèces de stomoxes capturées selon le biotope. 


\section{R. ZINGA KOUMBA et al. / Int. J. Biol. Chem. Sci. 8(1): 167-181, 2014}

Tableau 1 : Résultats globaux de la campagne de piégeage.

\begin{tabular}{|c|c|c|c|c|}
\hline $\mathbf{N}^{\circ}$ & Espèces de Tabanidae & Village & Forêt primaire & Effectifs total par espèce \\
\hline 1 & Ancala fasciata & 0 & 14 & 14 \\
\hline 2 & Chrysops dimidiatus & 4 & 8 & 12 \\
\hline 3 & Chrysops silacea & 119 & 7 & 126 \\
\hline 4 & Tabanus boueti & 3 & 41 & 44 \\
\hline 5 & Tabanus canus & 0 & 42 & 42 \\
\hline 6 & Tabanus congolensis & 20 & 15 & 35 \\
\hline 7 & Tabanus dilitius & 0 & 57 & 57 \\
\hline 8 & Tabanus obscurefumatus & 0 & 41 & 41 \\
\hline 9 & Tabanus obscurehirtus & 25 & 53 & 78 \\
\hline 10 & Tabanus par & 12 & 33 & 45 \\
\hline 11 & Tabanus secedens & 0 & 12 & 12 \\
\hline 12 & Tabanus socius & 2 & 41 & 43 \\
\hline \multirow[t]{2}{*}{13} & Tabanus taeniola & 7 & 67 & 74 \\
\hline & Espèces de Glossinidae & & & \\
\hline 14 & Glossina frezili & 3 & 81 & 84 \\
\hline 15 & Glossina fusca congolensis & 24 & 56 & 80 \\
\hline 16 & Glossina nashi & 26 & 162 & 188 \\
\hline 17 & Glossina palpalis palpalis & 74 & 112 & 186 \\
\hline \multirow[t]{2}{*}{18} & Glossina tabaniformis & 0 & 26 & 26 \\
\hline & Espèces de Stomoxyinae & & & \\
\hline 19 & Stomoxys niger bilineatus & 0 & 43 & 43 \\
\hline 20 & Stomoxys niger niger & 50 & 85 & 135 \\
\hline \multirow[t]{2}{*}{21} & Stomoxys omega & 15 & 120 & 135 \\
\hline & Effectifs totaux par biotope & 384 & 1116 & 1500 \\
\hline
\end{tabular}




\section{DISCUSSION}

Les résultats obtenus de cette étude constituent des données préliminaires sur les tabanidés, les glossines et les stomoxes dans cette partie de la province de l'Ogooué Ivindo, au Gabon. L'étude nous a permis d'établir une première liste des mouches hématophages présentes dans notre zone d'étude. Cette liste pourra ainsi servir de référence aux études complémentaires pour la lutte anti-vectorielle. Les pièges Nzi et Vavoua qui ont été utilisés au cours de ce travail présentent l'avantage de capturer le groupe cible (Stomoxes, glossine et tabanidés). Cependant, les faibles captures enregistrées pourraient s'expliquer par l'utilisation de ces deux types de piège. En effet, bien que l'efficacité de ces pièges pour la capture des stomoxes, tabanidés et glossines soit avérée (Djiteye, 1992; Djiteye 1994 ; Mihok et al., 1995b; Mihok, 2002 ; Gilles 2005 ; Gilles et al., 2007 ; Mavoungou et al., 2012), l'association de plusieurs types de piège (Nzi, grand Tetra, petit Tetra, Vavoua) et l'adjonction d'attractifs olfactifs comme l'octénol seul (Jaenson, 1991 ; Mihok et al., 1995b) ou en association avec des phénols (Mihok et al., 1995b) auraient peut-être permis d'augmenter les captures (Djiteye, 1992 ; Amsler and Fillerdier, 1994).

Les tabanidés représentent le groupe taxonomique le mieux étudié sur le site, du fait de leur abondance et du nombre d'espèces capturées. La répartition des espèces varie en fonction du biotope et toutes les espèces ne sont pas présentent dans les deux milieux; certaines espèces telles que A. fasciata, $T$. canus et $T$. secedens sont inféodées au milieu forestier. $\mathrm{Ce}$ résultat corrobore les observations faites dans la même localité qui a révélé une absence de ces espèces en milieu anthropisé (Mavoungou et al., 2012). Cette répartition varie aussi avec la saison. Ainsi, en saison des pluies, A. fasciata, T. dilitius, $T$. secedens et $T$. fusca congolensis n'ont été observés qu'en forêt, alors que $C$. dimidiatus n'a pas été capturé pendant cette saison. Cette répartition pourrait être liée également à la différenciation des paysages, la structure des milieux pouvant engendrer des micro-habitats particuliers plus ou moins favorables au développement de ces insectes. Les variations saisonnières jouent un rôle essentiel sur la validité des résultats. Aussi, des périodes de précipitations, représentées par la saison pluvieuse, peuvent être la principale cause d'évolution des effectifs d'insectes capturés. Le maximum d'espèces a été obtenu en saison des pluies aussi bien en forêt qu'en milieu anthropisé.

Contrairement aux tabanidés, la diversité spécifique des glossines est quasi uniforme sur le site d'étude. L'infestation d'un milieu par les glossines est conditionnée par la présence simultanée des facteurs environnementaux adéquats (température comprise entre $15{ }^{\circ} \mathrm{C}$ et $25{ }^{\circ} \mathrm{C}$, luminosité, humidité relative) et des vertébrés hôtes nourriciers (Foil and Gorham, 2000 ; Solano et al., 2010). Ces conditions sont retrouvées dans les deux biotopes prospectés et pourrait expliquer la distribution uniforme des glossines qui y est observée. Cependant, l'abondance des espèces reste hétérogène et varie significativement selon les biotopes et les saisons. En effet, en forêt tout comme au village, le maximum des captures a été observé en saison des pluies et le minimum en saison sèche. Aussi, cette répartition pourrait probablement être du fait de la variabilité des préférences trophiques des espèces de glossines. Alors que les espèces du sous-genre Nemorhina affichent un caractère alimentaire opportuniste et diversifié, se nourrissant invariablement sur l'homme et sur des animaux (Dagnogo et al., 1985 ; Laveissière et al., 1985), celles du sous-genre Austenina sont inféodées au gros gibier (Leak, 1999) très présent en forêt primaire.

Chez les Stomoxyinae, l'abondance et la diversité spécifique des populations varient en fonction des saisons et en fonction des biotopes. En effet, en saison des pluies, trois espèces de stomoxes ont été capturées avec des abondances variables en forêt alors qu'en milieu anthropisé deux espèces ont été capturées. Aussi, le maximum de capture a été observé en forêt primaire et le minimum au village. Ces résultats de forte abondance observés en saison des pluies corroborent ceux obtenus par Masmeatathip et al. (2006) et Mavoungou et al. (2008) qui ont montré que les stomoxes sont plus abondants en 
saison des pluies. De plus, le microclimat pourrait jouer aussi un rôle dans la répartition et l'abondance des différentes espèces de stomoxes. En étudiant la répartition des populations de l'araignée sociale Agelena consociata Denis à Makokou, Darchen (1978) avait enregistré des données thermométriques dans trois milieux : forêt, clairière et milieu ouvert. Il avait établi que la température moyenne était variable dans les trois milieux. En forêt, elle oscille entre $20{ }^{\circ} \mathrm{C}$ et $24{ }^{\circ} \mathrm{C}$ tandis qu'en milieu ouvert, elle se situerait entre 23 et $28{ }^{\circ} \mathrm{C}$.

$\mathrm{La}$ richesse spécifique de l'entomofaune en un lieu donné est définie par une plus ou moins grande diversité des espèces (Desquesnes et al., 2005). La forêt primaire est plus riche en espèces de diptères hématophages que le milieu anthropisé qui lui est adjacent. Cette diversité d'espèces serait en relation d'une part avec la stabilité du milieu forestier (omniprésence de l'humus, température plus ou moins constante, etc.) et d'autre part avec l'abondance de la grande faune, hôtes nourriciers des insectes hématophages (Broce et al., 2005). Les espèces identifiées au village ont été les espèces au caractère alimentaire anthropophile (Chrysops silacea) ou ubiquiste (G. palpalis palpalis). La présence de G. palpalis palpalis, vecteur de la maladie du sommeil, Chrysops silacea et de Chrysops dimidiatus, vecteurs de la loase à proximité des habitations et à l'intérieur de la forêt primaire évoque l'existence de risque sanitaire dans cette zone.

Les connaissances sur les insectes hématophages constituent un élément important dans la lutte anti-vectorielle, car ces insectes constituent un risque pour la santé humaine et animale. Mieux connaître la répartition des vecteurs, les risques de diffusion des pathogènes et leur évolution représente un enjeu majeur pour la mise en place d'une stratégie de contrôle de vecteurs en cas de transmission de pathogènes.

\section{REMERCIEMENTS}

Ce travail a été réalisé grâce à l'appui financier, institutionnel et logistique de l'Union Européenne via le projet ACPFORENET, l'Institut de Recherche en
Ecologie Tropicale (IRET) et le Laboratoire d'Ecologie Vectorielle (LEV). Nous remercions Nicolas EMBA YAO et Stéphane ESSASOU BADJI pour leur aide technique sur le terrain.

Nos sincères remerciements sont dirigés à l'adresse du Professeur Gérard DUVALLET, de monsieur Ephrem NZENGUE, Patrick ONDO OKAMA et Karl Bader HENGA BOTSIKA BOBE dont les remarques, suggestions et corrections ont permis d'améliorer ce manuscrit.

\section{REFERENCES}

Broce AB, Hogsette J, Paisley S. 2005. Winter feeding sites of hay in round bales as major developmental sites of Stomoxys calcitrans (Diptera: Muscidae) in pastures in spring and summer. Journal of Economic Entomology, 98(6): 23072312.

Baldacchino F, Muenworn V, Desquesnes Marc, Desoli F, Charoenviriyaphap T, Duvallet G. 2013. Transmission of pathogens by Stomoxys flies (Diptera, Muscidae): a review. Parasite, 26(20): p13.

Brunhes J, Cuisance D, Geoffroy B, Hervy JP. 1998. Les glossines ou Mouches Tsétsé. Logiciel d'Identification et d'Enseignement. Editions ORSTOM, Montpellier, France.

Dagnogo M, Lohuirignon K, Gouteux JP. 1985. Comportement alimentaire des populations péridomestiques de Glossina palpalis (Robineau-Desvoidy) et Glossina tachinoides Weswood du domaine guinéen de Côte d'Ivoire. Cahier ORSTOM, série Entomologie Médicale et Parasitologie, 23: 3-8.

Darchen R. 1978. Les populations d'Agelena consociate Denis, araignée sociale, dans la forêt primaire gabonaise. Leur répartition et leur densité. Annales de Sciences Naturelles, Zoologie, Paris, 14(2): 19-26.

Desquesnes M, Dia ML, Acapovi GL, Yoni W, Foil L, Pin R. 2005. Les Vecteurs Mécaniques des Trypanosomoses Animales : Généralités, morphologie, Biologie, Impacts et Contrôle. 
Identification des Espèces les plus Abondantes en Afrique de l'Ouest. Editions Cirdes, Imprimerie de l'Avenir : Burkina Faso ; 68 p.

Djiteye A. 1992. Aperçu sur l'efficacité comparative de différents pièges et odeurs contre les mouches piqueuses (Diptera : Tabanidae et Muscidae) d'importance vétérinaire. In Premier séminaire International sur les Trypanosomoses Animales non Transmises par les Glossines, Annecy (France), 14-16 octobre 1992.

Djiteye A. 1994. Efficacité comparée des différents types de pièges et / ou associations d'odeurs sur les tabanidés, stomoxes et glossines présentes en zone soudano-guinéenne (Mali) G. $m$. submorsitans, G. tachinoüdes, G. $p$. gambiensis. Résumé Rapport d'étape, LCV de Bamako, Mali.

Foil LD. 1989. Tabanids as vectors of disease agents. Parasitology Today, 5(3) : 88-96.

Foil LD, Gorham JR. 2000. Mechanical transmission of disease agents by arthropods. In Medical Entomology, Eldridge BF, Edman JD (eds). Kluwer Academic Publishers: Dordrecht, the Netherlands; 461-514.

Jaenson TG, Dos Santos RC, Hall DR. 1991. Attraction of Glossina longipalpalis (Diptera: Glossinidae) in Guinea-Bissau to odorbaited biconical traps. Journal of Medical Entomology, 28: 284-286.

Garros C, Gilles J, Duvallet G. 2004. Un nouveau caractère morphologique pour distinguer Stomoxys calcitrans et S. niger (Diptera : Muscidae). Comparaison de populations de l'île de La Réunion. Parasite, 11: 329-332.

Georgelin. 1923. Maladie endémiques et endémo-épidémiques constatées au Gabon pendant l'année 1921-1922. Annales de Médecine et de Pharmacie Coloniales, 21: 209-15.

Gilles J. 2005. Dynamique et génétique des populations d'insectes vecteurs. Les stomoxes, Stomoxys calcitrans et Stomoxys niger niger dans les élevages bovins réunionnais. Thèse de doctorat,
Université de La Réunion, St-Denis, 140 p.

Gilles J, David JF, Duvallet G, de La Rocque S, Tillard E. 2007. Efficiency of traps for Stomoxys calcitrans and Stomoxys niger on Reunion Island. Medical and Veterinary Entomology, 21: 65-69.

Koné N, N'Goran EK, Sidibe I, Kombassere AW, Bouyer J. 2011. Spatio-temporal distribution of tsetse and other biting flies in the Mouhoun River basin, Burkina Faso. Medical and Veterinary Entomology, 25: 156-168

Laveissière C, Couret D, Staak C, Hervouët JP. 1985. Glossina palpalis et ses hôtes en secteur forestier de Côte d'Ivoire. Relation avec l'épidémiologie de la trypanosomiase humaine. Cahier ORSTOM, série Entomologie Médicale et Parasitologie, 23(4) : 297 - 303.

Laveissière C, Grébaut P. 1990. Recherche sur les pièges à glossines (Diptera : Glossinidae). Mise au point d'un modèle économique: le piège «Vavoua». Tropical Medicine and Parasitology, 41(2): 185-192.

Leak SGA. 1999. Tsetse biology and ecology. Their role in the epidemiology and control of trypanosomiasis. CABI Publishing: New York; 568p.

Leclercq M. 1967. Contribution à l'étude des Trypetidae (Diptera) paléarctiques et de leurs relations avec les végétaux. Bulletin de Recherche Agronomique de Gembloux, 2(1) : 64-105.

Masmeatathip R, Gilles J, Ketavan C, Duvallet G. 2006. First survey of seasonal abundance and daily activity of Stomoxys $\mathrm{s}$ pp. (Diptera: Muscidae) in Kamphaengsaen campus, Nakornpathom Province Thailand. Parasite, 13: 245-250.

Mavoungou JF, Jay-robert P, Gilles J, Atsame Edda A, Duvallet G. 2008. Ecologie des stomoxes (Diptera: Muscidae) au Gabon. I. Premier inventaire dans différentes zones écologiques. Parasite, 15: 27-34.

Mavoungou JF, Makanga B, Acapovi YG, Desquesnes M, M'batchi B. 2012. Chorologie des Tabanidae (Diptera) dans la réserve de Biosphère IPASSA- 
MAKOKOU (GABON) en saison des pluies. Parasite, 19: 165-171.

Mihok S, Kangethe EK, Kamau GK. 1995. Trials of Traps and Attractants for Stomoxys spp. (Diptera, Muscidae). Journal of Medical Entomology, 32(3): 283-289.

Mihok S. 2002. The development of a multipurpose trap (the Nzi) for tsetse and other biting flies. Bulletin of Entomological Research, 92: 385-403.

Milleliri JM, Kohagne TL, Mengue MP, Louis FJ. 2009. La maladie du sommeil au Gabon. Cent cinquante ans d'une histoire contrastée. 1861-2008. Sciences et Médecine d'Afrique, 2: 119-132.

Muzari MO, RE Jones, LF Skerratt, TL Duran. 2010. Feeding success and trappability of horse flies evaluated with electrocuting nets and odour-baited traps. Veterinary Parasitology, 171: 321-326.

Oldroyd H. 1952. The Horse Flies (Diptera: Tabanidae) of the Ethiopian Region (vol 1). British Museum (Natural History): London; $226 \mathrm{p}$.

Oldroyd H. 1954. The Horse Flies (Diptera: Tabanidae) of the Ethiopian Region (vol 2). British Museum (Natural History): London; 341p.

Oldroyd H. 1957. The Horse Flies (Diptera: Tabanidae) of the Ethiopian Region (vol 3). British Museum (Natural History): London; $489 \mathrm{p}$.

Oldroyd H. 1973. Tabanidae in Smith: Insects and other Arthropods of Medical Importance. British Museum. (Natural History): London ; 195-202.

Pollock JN. 1992. Manuel de Lutte Contre la Mouche Tsé-Tsé. Biologie, Systématique et Répartition des Tsé-Tsé (vol 1). FAO : Rome ; $310 \mathrm{p}$.

Rodhain FS, Perez C. 1985. Précis d'Entomologie Médicale et Vétérinaire. Edition Maloine : Paris, France ; 648p.

Solano P, Bouyer J, Itard J, Cuisance D. 2010. The cyclical vectors of trypanosomis. Infectious and Para Diseases of Livestock., 13: 155-183.

TEREA. 2010. Rapport d'inventaire d'aménagement. Concession de Makokou, (tome 1). Tech. rep., Olam, Libreville, Gabon.

Toure FS, Mavoungou E, Deloron P, Egwang TG. 1999. Analyse comparative de deux méthodes diagnostiques de la loase humaine: sérologie IgG4 et PCR nichée. Bulletin de la Société de Pathologie Exotique, 92: 167-170.

Toure FS, Kassambara L, Williams T, Bain O, Millet P. 1998. Human occult loasis: improvement in diagnostic sensitivity by the use of polymerase chain reaction. American Journal of Tropical Medecine and Hygiene, 59: 144-149.

Van Hoegaerden M, Chabaud B, Akue JP, Ivanoff B. 1987. Filariasis due to Loa loa and Mansonella perstans: distribution in the region of Okondja, Haut Ogooué Province, Gabon, with parasitological and serological follow-up over one year. Transaction of the Royal Society of Tropical Medecine and Hygiene, 81: 441446.

Zumpt F. 1973. Diptera: Muscidae; Taxonomy and biology of species. In The Stomoxyinae Biting flies of the World. Gustav Fischer Verlag: Stuttgart, Germany; 20-97. 